\title{
Editorial
}

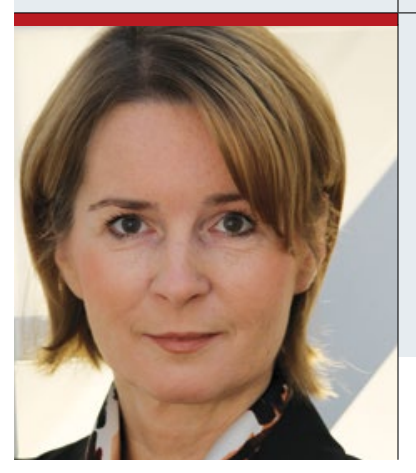

Katja Kupfer-Geißler

Chefredaktion

katja.kupfer@springer.com

facebook.com/springerpflege

\section{Chancen nutzen}

F ür mich ist der Bericht von Professor Wolfgang Rösch (Seite 29) harter Tobak. Die Kinderurologische Klinik der KUNO Kinderklinik St. Hedwig in Regensburg, so berichtet er, konstatiert derzeit eine Zunahme der Behandlungsfälle durch Flüchtlingskinder um bis zu 3\% bei einzelnen Erkrankungen. Das scheint doch gar nicht so viel zu sein? Doch wenn man liest, mit welchen Diagnosen das Team dort zu tun hat, dann bin ich froh über jedes Kind, das es bis zum ihm geschafft hat. Denn es geht hier nicht um Bagatellerkrankungen, es geht um massive Verletzungen der Kinder beispielsweise durch Tretminen oder Beschneidungen.

In Regensburg hat man sich der Aufgabe gestellt, die Flüchtlingskinder und ihre Angehörigen aufzufangen und ihnen zu helfen. Dass dies gelungen ist, zeigt die Auszeichnung der Klinik mit dem Bayerischen Gesundheits- und Pflegepreis 2017. Bei uns ist der Beitrag von Prof. Rösch nicht nur Bestandteil des PflegeKollegs „Kultursensibel pflegen“.
Auch in der Reportage (Seite 42) kommen die Regensburger zu Wort. Hier berichten sie, dass sie nicht nur den Flüchtlingen helfen, sondern auch ihren Mangel an geeignetem Nachwuchs ein wenig lindern konnten - also, win-win.

In diesem Sinn wünsche ich Ihnen mit dem gesamten Springer Pflege-Team eine frohe Weihnachtszeit und

\section{viel Freude beim Fortbilden mit HEILBERUFE} Ihre

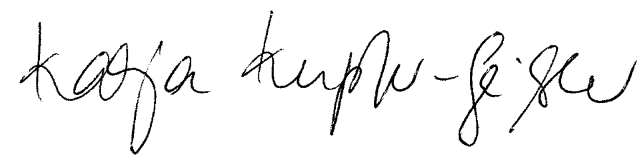

Anregungen, Wünsche, Fragen oder Lob? Schreiben Sie uns: katja.kupfer@springer.com

\section{ANTIBIOTIKA-VERBRAUCH}

\section{ROBERT KOCH INSTITUT}

\section{Neu: Antibiotika-Verbrauchs-Surveillance}

Das Robert Koch-Institut bietet seit Ende November mit der Antibiotika-Verbrauchs-Surveillance (https:// avs.rki.de) erstmals allen Krankenhäusern und anderen Gesundheitseinrichtungen die Möglichkeit, ihren Antibiotikaverbrauch mit Referenzdaten zu vergleichen. Die Daten können über eine interaktive Datenbank abgerufen werden. In Zusammenarbeit mit dem Nationalen Referenzzentrum für die Surveillance von nosokomialen Infektionen (NRZ) hat das RKI die Antibiotika-Verbrauchs-Surveillance (AVS) für den stationären Sektor aufgebaut. Bisher haben sich mehr als 300 Krankenhäuser und Rehabilitationskliniken angemeldet, 190 Einrichtungen liefern Daten. Neben der Verbrauchs-Surveillance führt das RKI auch eine Antibiotika-Resistenz-Surveillance (ARS) durch, mit der Kliniken und niedergelassene Ärzte die Resistenzentwicklung vor Ort verfolgen und ihre Verordnung anpassen können (https://ars.rki.de). 